\title{
Pinning down viral proteins: a new prototype for virus-host cell interaction
}

\author{
Yoshitsugu Kojima ${ }^{1,2}$ and Akihide Ryo ${ }^{1 *}$ \\ Department of Microbiology, Yokohama City University School of Medicine, Yokohama, Kanagawa, Japan \\ 2 Japan Foundation for AIDS Prevention, Tokyo, Japan
}

Edited by:

Hironori Sato, National Institute of Infectious Diseases, Japan

\section{Reviewed by:}

Masaru Yokoyama, National Institute of

Infectious Diseases, Japan

Takao Masuda, Tokyo Medical and

Dental University, Japan

Kazushi Motomura, National Institute

of Infectious Diseases, Japan

\section{*Correspondence:}

Akihide Ryo, Department of

Microbiology, Yokohama City University

School of Medicine, 3-9 Fuku-ura,

Kanazawa-ku, Yokohama, Kanagawa

236-0004, Japan

e-mail:aryo@yokohama-cu.ac.jp
Pin 1 is an enzyme that specifically catalyzes the cis-trans isomerization of phosphorylated serine/ threonine-proline (pSer/Thr-Pro) motif in its substrate proteins. Recent studies demonstrate that stability of several viral proteins is regulated by phosphorylation-dependent prolyl-isomerization by a host factor Pin1. Pin1 is now positioned as an important modulator of the molecular crosstalk between virus and host cells and could be a unique target for anti-virus therapy. This new type of post-translational modification by Pin1 might be involved in the regulation of other viral proteins.

Keywords: phosphorylation, prolyl-isomerization, protein stability, Pin1
Post-translational modifications of proteins are major regulatory events in many cellular processes such as cell proliferation, differentiation, and cell death. In particular, protein phosphorylation is a major mode of post-translational modifications and an important regulatory event for many cellular processes by modulating intracellular signaling pathways (Hunter, 1995). It has been reported that phosphorylated proteins are subsequently subjected to a new type of "post-phosphorylation" regulation by a peptidylprolyl isomerase Pin1. Pin 1 is a peptidylprolyl cis-trans isomerase and only binds to serine or threonine residue immediately preceding a proline residue (Ser/Thr-Pro). After binding to the motif, Pin1 dynamically changes the conformation of target proteins via cis-trans isomerization of the peptide bonds. Such conformational changes have profound effects on the function of substrate proteins by modulating their catalytic activity, protein-protein interaction, sub-cellular localization, and protein stability (Ryo et al., 2003; Lu et al., 2007). With the diverse physiological roles of Pin1, it has been shown that Pin 1 is linked to the etiology of several diseases that include cancers, Alzheimer's disease and immune diseases (Lu and Zhou, 2007). In addition to this, recent studies demonstrate that the stability and function of several viral proteins are also regulated by phosphorylation-dependent Pin1-mediated prolyl-isomerization (Figure 1).

Pang et al. (2007) firstly identified Pin 1 as a novel binding partner for the hepatitis B virus X protein ( $\mathrm{HBx}$ ), a viral encoding oncoprotein. The interaction appears to have significant effects on the stability and pro-tumorigenic activity of the viral protein (Pang et al., 2007). Pin1 overexpression was found to be related to HBx expression in HBV-related tumors. Pang et al. (2007) confirmed that Pin1 binds HBx at the specific phosphorylated the Ser41-Pro motif. This interaction was shown to be inhibited by the mitogen-activated protein kinase/extracellular signal-regulated kinase (MEK) inhibitor, suggesting the possible role of mitogen-activated kinase (MAPK) family in the phosphorylation of the Ser41-Pro motif. Pin1 overexpression was shown to increase the protein stability of $\mathrm{HBx}$ as well as $\mathrm{HBx}$ mediated transactivation. Concomitant expression of Pin 1 and $\mathrm{HBx}$ in the non-tumorigenic human hepatocyte cell line MIHA led to a synergistic increase in tumor growth. Moreover, in hepatocellular carcinoma Hep3B cells with suppressed Pin 1 expression, HBx-mediated tumor growth in nude mice was abrogated. These results together indicate that Pinl enhance hepatocarcinogenesis in HBV-infected hepatocytes by activating both stability and function of $\mathrm{HBx}$.

The second target for Pin1-viral protein interaction is HTLV-1 Tax. Two groups have reported the functional interaction between HTLV-1 Tax oncoprotein and Pin1 (Jeong et al., 2009; Peloponese Jr. et al., 2009). Pin 1 is highly expressed in adult T cell leukemia (ATL) cells expressing Tax protein and forced expression of Pin 1 in turn increases the Tax protein expression. Pin 1 prolonged the protein stability of Tax by suppressing the ubiquitination and subsequent lysosomal degradation of Tax. Pin 1 interacts with phosphorylated Tax on its Ser160-Pro motif. On the other hand, a Pin1 inhibitor Juglone suppressed cell proliferation of the Tax-expressing T cell line. Thus, Pin1 plays a supporting role in Tax-mediated cell transformation in the post-translational regulation of Tax. The targeting of Pin1 may offer a new insight into the pathogenesis of HTLV-1 related diseases such as ATL.

A recent study has demonstrated that HIV integrase (IN) is a new target for Pin 1 . Indeed, the protein stability HIV IN was found to be regulated by phosphorylation-dependent Pin1-catalyzed prolyl-isomerization (Manganaro et al., 2010). Exogenously transfected HIV IN can associate with Pin1. Furthermore, such interaction is dependent on phosphorylation of HIV IN, specifically on the Ser57-Pro motif, which can be phosphorylated by host kinase c-Jun N-terminal kinase (JNK). Importantly, this interaction has indeed profound functional significance. HIV IN is a protein with a short half-life, but its steady-state levels were shown to significantly 


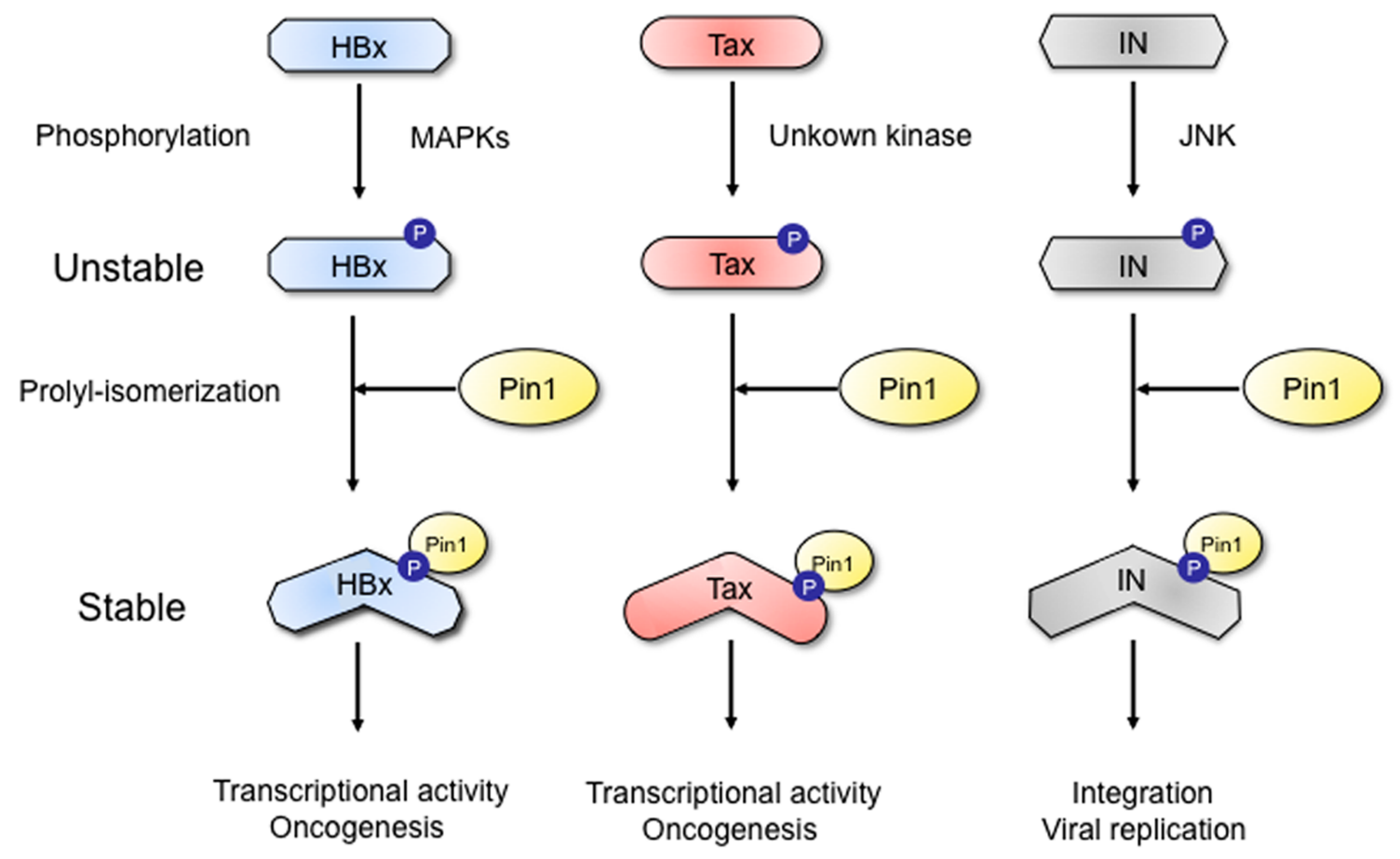

FIGURE 1 |A novel post-phosphorylation regulatory mechanism for viral protein stability. The phosphorylation of viral proteins (HBV HBx, HTLV-1 Tax, and HIV IN) by the proline-directed kinases (JNK, MAPK etc.) generates binding modules for Pin1. Subsequent prolyl-isomerization by Pin1 induces conformational changes and thereby enhances the viral protein stability.

increase during Pin1 interaction. In addition to regulating protein stability, Pin 1 can concomitantly enhance the activity for HIV IN thereby facilitating the HIV-1 proviral integration into the host cell genome. These concerted activities of Pin1-dependent prolylisomerization can in turn lead to efficient HIV-1 replication. Since the lack of these modifications restricts viral infection, Pin 1 could be an intriguing target for anti-HIV therapy.

In conclusion, these observations are of special relevance to Pin 1 for the natural course of viral replication via modulating the stability and function of viral proteins. Because it is quite likely that other viral proteins in different viral species could also be Pin1 targets, future studies for Pin1-viral protein interaction would

\section{REFERENCES}

Hunter, T. (1995). Protein kinases and phosphatases: the yin and yang of protein phosphorylation and signaling. Cell 27, 225-236.

Jeong, S. J., Ryo, A., and Yamamoto, $\mathrm{N}$. (2009). The prolyl isomerase Pin 1 stabilizes the human T-cell leukemia virus type 1 (HTLV-1) Tax oncoprotein and promotes malignant transformation. Biochem. Biophys. Res. Commun. 381, 294-299.

Lu, K. P., Finn, G., Lee, T. H., and Nicholson, L. K. (2007). Prolyl cistrans isomerization as a molecular timer. Nat. Chem. Biol. 3, 619-629.

Lu, K.P., and Zhou, X.Z. (2007). The prolyl isomerase PIN1: a pivotal new twist in phosphorylation signalling and disease. Nat. Rev. Mol. Cell Biol. 8, 904-916.

Manganaro, L., Lusic, M., Gutierrez, M. I., Cereseto, A., Del Sal, G., and Giacca, M. (2010). Concerted action of cellular JNK and Pin1 restricts HIV-1 genome integration to activated CD4+ T lymphocytes. Nat. Med. 16, 329-333.

Pang, R., Lee, T. K., Poon, R. T., Fan, S. T., Wong, K. B., Kwong, Y. L., and Tse, E. (2007). Pin1 interacts with a specific serine-proline motif of hepatitis $B$ virus $\mathrm{X}$-protein to enhance hepatocarcinogenesis. Gastroenterology 132, 1088-1103.

Peloponese, J. M. Jr., Yasunaga, J., Kinjo, T., Watashi, K., and Jeang,

shed new light on molecular etiology of virus-host cell interaction. Furthermore, these studies may also have therapeutic implications of Pin1 inhibition in viral infection. With specific Pin1 inhibitors being developed, the application of such molecules in combination with current antiviral therapies could change the course of viral replication and prevent the development of viral pathogenicities.

\section{ACKNOWLEDGMENTS}

Yoshitsugu Kojima is a research resident of the Japan Foundation for AIDS prevention. This work was in part supported by grants from the Takeda Science Foundation and the Japanese Ministries of Education, Culture, Sports, Science and Technology to Akihide Ryo.

K. T. (2009). Peptidylproline cistrans-isomerase Pin 1 interacts with human T-cell leukemia virus type 1 tax and modulates its activation of NF-kappaB. J. Virol. 83, 3238-3248.

Ryo, A., Liou, Y. C., Lu, K. P., and Wulf, G. (2003). Prolyl isomerase Pin1: a catalyst for oncogenesis and a potential therapeutic target in cancer.J. Cell. Sci. 116, 773-783.

Conflict of Interest Statement: The authors declare that the research was conducted in the absence of any commercial or financial relationships that could be construed as a potential conflict of interest.
Received: 24 July 2010; paperpending published: 30 July 2010; accepted: 09 August 2010; published online: 09 September 2010.

Citation: Kojima Yand Ryo A (2010) Pinning down viral proteins: a new prototype for virus-host cell interaction. Front. Microbio. 1:107. doi:10.3389/fmicb.2010.00107

This article was submitted to Frontiers in Virology, a Specialty of Frontiers in Microbiology.

Copyright $\odot 2010$ Kojima and Ryo. This is an open-access article subject to an exclusive license agreement between the authors and the Frontiers Research Foundation, which permits unrestricted use, distribution, and reproduction in any medium, provided the original authors and source are credited. 